\title{
3D-Printed Personalized Titanium Implant Design,Manufacturing and Verification for Bone Tumor Surgery of Forearm
}

\author{
Kwun Mook Lim ${ }^{1}$, Jong Woong Park ${ }^{2}$, Sung Jun Park ${ }^{1}$ and Hyun Guy Kang*2 \\ ${ }^{1}$ Department of Mechanical Engineering, Korea National University of Transportation, Korea
}

${ }^{2}$ Orthopaedic Oncology Clinic, Specific Organs Cancer Branch, National Cancer Center, Korea

Received: 眥: October 10, 2018; Published: 制: October 25, 2018

*Corresponding author: Hyun Guy Kang, Orthopaedic Oncology Clinic, Specific Organs Cancer Branch, Research Institute and Hospital, Graduate School of Cancer Science and Policy, National Cancer Center 323 Ilsan-ro, Ilsandong-gu, Goyang-si Gyeonggi-do, 10408, Republic of Korea

\begin{abstract}
The 3D printing implants are currently being used to reconstruct various parts of the bone tumor area other than maxillofacial surgery area. Thanks to 3D printing implants, patients can remain their anatomic function of bone through reconstruction surgery, which was impossible before since patients became handicapped. The segmental prosthesis of the long bone needs a special design for fixation with normal bone as well as maintaining the joints and mechanical stability. In this preliminary report one can find procedures of test surgery on volunteer patient which include design, manufacturing process, inspection, and mechanical test procedures. The patient underwent reconstruction surgery using 3D printing metal implant for both forearm bones. By using 3D printing metal prosthesis for reconstruction of the bone defect, surgeons were able to shorten duration of the surgery and maintained the anatomical reconstruction for the all bones of patient's body.
\end{abstract}

Keywords: Forearm; Bone Tumor; 3D Printed Implant

\section{Introduction}

The treatment of primary malignant bone tumors has progressed over decades and this has led to the success of limb salvage procedures in approximately $85 \%$ of cases [1-9]. As far as the limb salvage procedures are concerned, an endoprosthetic replacement has been regarded as the standard reconstruction technique after wide excision for bone sarcomas in musculoskeletal oncology centers [10]. However, commercially available modular type of the prosthesis is supplied only for commonly used replacements such as the femur, tibia and humerus. As for reconstruction of uncommon bones, a custom- made prosthesis was one of available surgical options, but the problem was that it took a long time to fabricate and it was expensive. Frankly, the 3D printing implant is applicable to any bones of all sites. Furthermore, it requires relatively short fabrication time and is cheaper than a conventional custom-made implant. The manufacturing of specific 3D implant is composed of five stages;

a) First, obtaining the 3D model of the patient by receiving the DICOM files (DICOM, Digital Imaging and Communications in Medicine) from the hospital.

b) Second, designing the implant and cutting guide. c) Third, printing implant and cutting guide using each 3D printer.

d) Fourth, post-processing process.

e) Fifth, processing implant's inspection and cleaning.

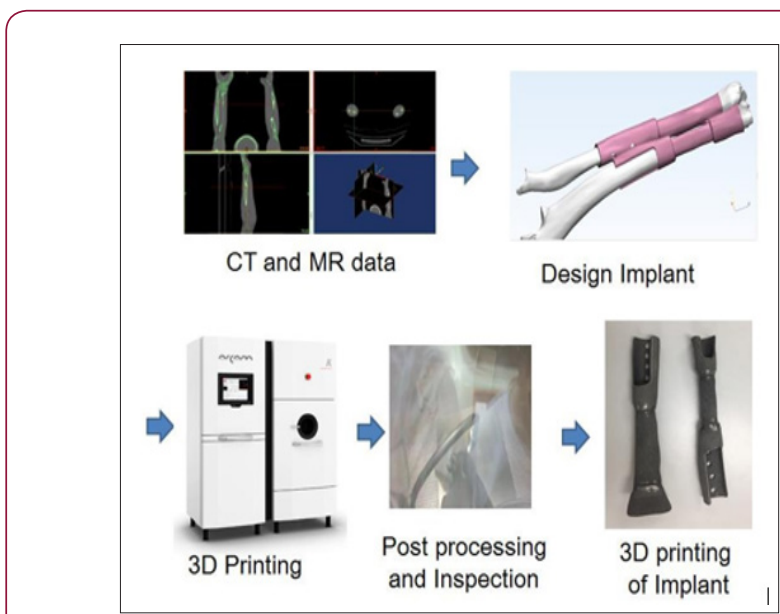

Figure 1: Overview of customized tumor implants. 
This study aims to demonstrate the whole process of 3D printing titanium tumor implants including implant design, manufacturing, and performance evaluation. In order to deliver the outcomes, our team would like to report a representative case of reconstructive surgery with 3D printing prosthesis for both forearm bone while preserving the adjacent joints of the forearm (Figure 1).

\section{Materials and Methods}

\section{Patient Information}

The patient is a 40 -year-old man, working at local clinic as an office worker. He had a right hand numbness and limitation in the range of motion of forearm rotation. He had no medical problem and denied any medications. His right radial head was dislocated and limited motion of elbow flexion and forearm rotation since childhood due to uncertain accident. In addition, he had undergone orthopedic surgery using external fixator for the right forearm 5 years ago. After the surgery, recovery and rehabilitation were done and he used his forearm with limited forearm motion. However, limitation of range of motion of the forearm had been getting more severe recently and started imaging for the forearm. The forearm rotation was possible from $45^{\prime}$ supination to $30^{\prime}$ pronation at the $1^{\text {st }}$ visit for our institute. Our team found out abnormal results of plan radiograph, ultrasonography and MRI of right forearm soft tissue and bone. The plain radiographs showed the large bone (radius) defect which was assumed area of pinning site of external fixator and slight bony erosion of ulna. The MRI showed the big soft tissue mass which was semicircular wrap of radius and ulnar (Figure 2).
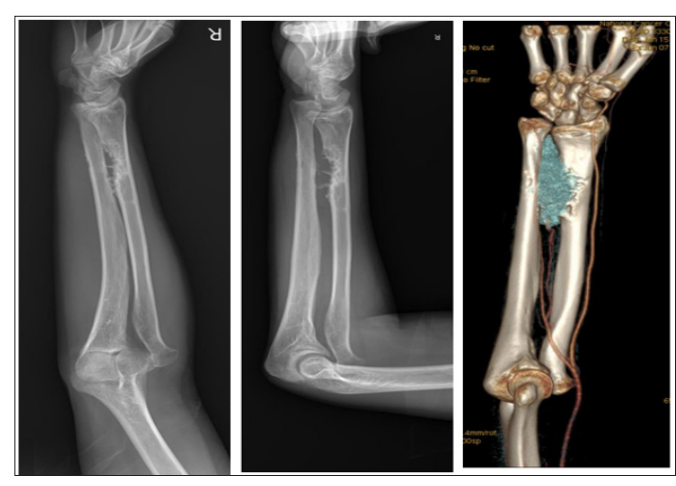

Figure 2: A preoperative forearm photo of X-ray AP view (left), A preoperative forearm photo of X-ray lateral view (middle), A preoperative forearm photo of CT reconstruction(right).

The sono-guided core needle biopsy resulted desmoplastic fibroma that was locally aggressive intermediate tumor and needed wide excision to prevent local recurrence the soft tissue tumor from originate radius extended adjacent wrist joint. The distant metastasis was not demonstrated on the bone scan and PET/CT examination. In order to maximally maintain the anatomical configuration of both forearm bones, while preserving adjacent joints including the wrist joint and the distal radio-ulnar joint, our team decided to reconstruct the bone defect with 3D-printed implants. Forearm rotation movement is very complicated interaction between both forearm bones, and greatly limited by very slight anatomical ab- normalities and remaining distal radius and ulnar fragment were expected too small to fixate by conventional methods using screws and plates. Therefore, we planned limb salvage surgery with skeletal reconstruction by patient-specific 3D printed radius and ulnar implant that were designed by mirror image of both forearm bones of the normal side. For the fixation between implants and remained host bones, each part was designed to be customized considering the direction of approach, safe screw trajectory, and enough fixing force.

\section{Virtual Surgical Simulation}

Axial CT images of the forearm were CT scanner and the slices of $0.25 \mathrm{~mm}$ thickness. The CT images in DICOM format were then imported into medical image analysis software (MIMICS 17.0, Materialise NV, Leuven, Belgium). The CT image data sets were then reformatted into coronal and sagittal views in addition to the original axial views. A gray-valued based segmentation of the CT images excluded the soft tissue and a 3D model of the affected forearm was generated for surgical simulation. As MR images showed the same tumor involvement as on the CT images, the extent of the tumor was outlined from the CT images and its volume was extracted. Afterwards, a 3D bone-tumor model was created. All the reformatted 2D images and 3D models were analyzed for preoperative surgical planning. A partial forearm resection with a $10-\mathrm{mm}$ safe margin was virtually simulated. The working file of the surgical simulation was then forwarded to the implant engineer (MEDYSSEY R\&D Center, Jaechoen, Korea).

\section{Design of the Patient-Specific Forearm Implant}

The most important considerations of tumor implant device in orthopedic oncology surgery are to confirm strength of the part of bone replacing and to design the connection part between the implant and remaining bones so that the implant was easily and firmly attached to the bone. Moreover, anatomical reconstruction is common important issue for specific skeletons including both forearm bones to gain maximal function. For the designing step, cooperated with surgeon for his clinical knowledge or/else information of surgical approach and engineer owing to his knowledge of mechanical and structural property. The model created in Mimics was exported to 3-Matic (Materialize, Belgium) for further processing to isolate the forearm and to construct the final implant model. 3-Matic provides with a means of error corrections, where the DICOM data model can be checked for issues such as multiples shells and inverted normal, such that any produced design would be fit for digital manipulation and the final 3D printing. For the patient with the bone tumor on the both forearm bones, the entire shaft of the implant was basically modeled to the same size as the original bone(Figure 3).

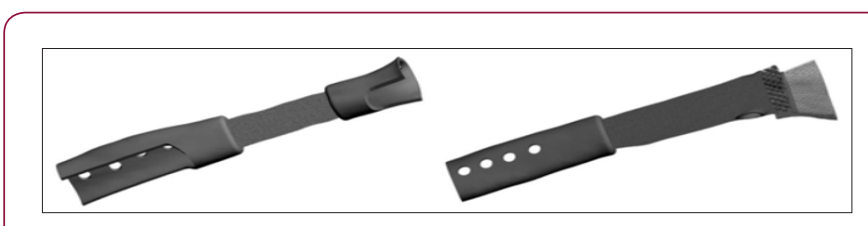

Figure 3: CAD images of forearm from the STL files. (Ulna and Radius). 
For the connection part between the implant and remaining host bones, each part needed different designs according to circumstances of the junctions. Basically, common designs for the connection part were a metal plate for screw fixation and a cylindrical cuff skirt to insert remaining long bone segment. The cuff skirt was a circumferential metal plate of ring shape, which allowed a remaining host bone to be inserted. The thickness of the metal plates and cuff skirts was $3 \mathrm{~mm}$ and the diameter of the screw holes was 3.5 $\mathrm{mm}$. For proximal shaft of radius and ulna, a complex of metal plate and cuff skirt was used, because the host bone segment to connect to the implant had cylindrical shape. The lengths of the cuff skirt part were not exceeding $1 \mathrm{~cm}$. While, for the distal radius and ulna, the remaining host bone remarkably became wider toward the distal direction. Therefore, for those distal parts, only metal plates were used. The dorsal side of the distal radial part of the implant has been emptied to prevent tendon abrasion and it had two screw holes through the implant in the shape of $\mathrm{X}$ shape. In order to enhance bone incorporation, both ends of the radial and ulnar implants, which contacted to host bones, had porous structure.

\section{Implant 3d Printing and Post Processing}

The final implant was printed using electron beam melting (EBM) within an ARCAM A1 (Arcam AB, Sweden) using medical grade titanium (Ti6Al4V-ELI per ASTM 136). The ARCAM EBM A1 machines are based on Electron Beam Melting (EBM). During the EBM process, the electron beam melts metal powder layer-by-layer to build the implant. The vacuum environment in the EBM machine maintains the chemical composition of the material and provides an excellent environment for building parts in reactive materials such as titanium alloys. The high power of the Electron Beam ensures a high rate of deposit and an even temperature distribution within the part. The results trigger full melting of the metal powder and high strength properties of the material. The EBM machine Titanium Ti6Al4V ELI (Grade 23) is a gas-atomized powder with a particle size between 45 and 100 microns. This limit on the minimum particle size ensures safe handling of the powder. Ti6Al4V-ELI has numerous applications in the medical industry. The biocompatibility of Ti6Al4V-ELI is excellent, especially when direct contact with tissue or bone is required. The final 3D printing implant was cleaned ultrasonically. Subsequently, the sterilization with EO gas was performed in the hospital (Figures 4 \& 5).

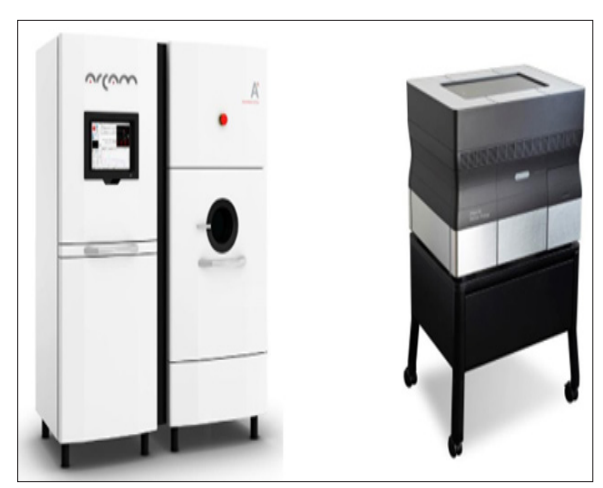

Figure 4: 3D printing Machine (left : ARCAM A1 for Metal Implant, Right: OJBECT 30 Prime for Plastic model).

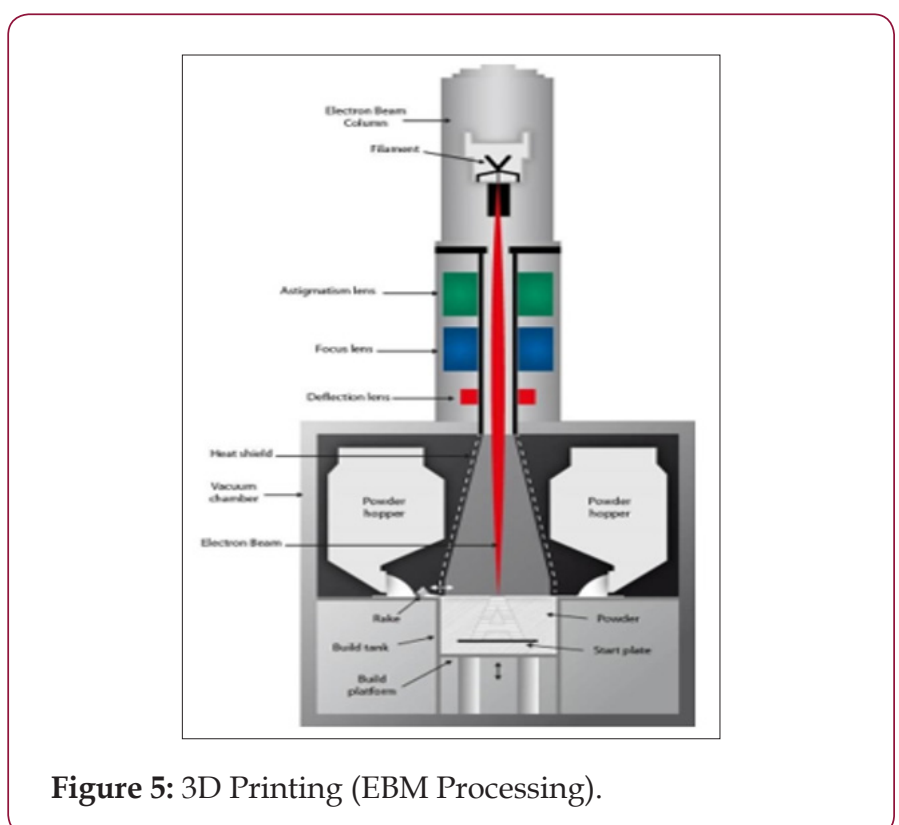

Performance Evaluated of the 3D printed forearm implant

The mechanical testing was performed at porous shaft part, which links the skirt with plate of titanium forearm implants using 3D printing. All mechanical testing was performed another set of the implants which were same as the implants used in the actual surgery. Five samples of each Ulna and Radius were subjected to 3 -point bending using a $25 \mathrm{~mm}$ displacement control per minute and span of distance was $25 \mathrm{~mm}$ using ASTM F382 (Metallic Bone Plate Material Bend Test). After obtaining each test results (Maximum load), calculated the 3-point bending strength as shown in the following formula; (Maximum load $\mathrm{x}$ distance) / 2, and compared it with the experimental data of the patient's body, specifically, ulna and radius bone. Despite the shaft part was confirmed by the actual mechanical test, but the confirmation of the plate and the fixed part of the plate and skirt was more necessary. Therefore, we conducted comparative analysis with the existing commercial with widely used trauma plate. Commercial ulna and radius trauma plate has a thickness of about $2.0 \sim 2.5 \mathrm{~mm}$ and usually is made of unalloyed commercially pure titanium grade 2 to 4 , the screws with $2.0 \sim 3.0 \mathrm{~mm}$ size are used (Figure 6).

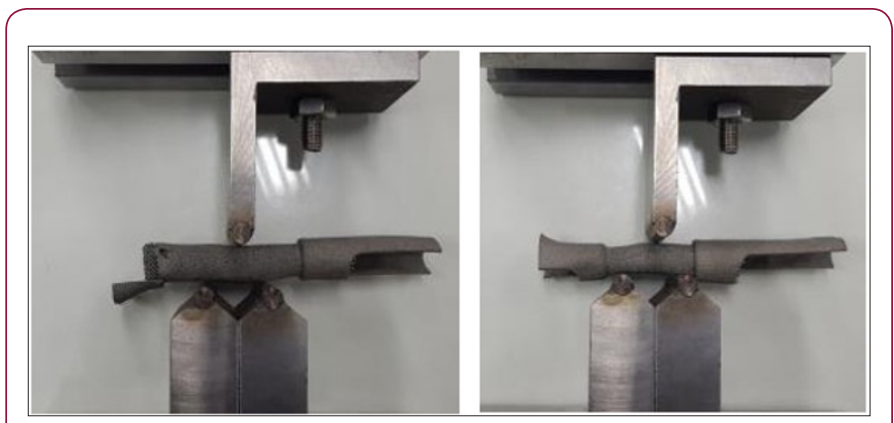

Figure 6: Mechanical testing Jig (ulna and radius part).

In this case, 3D printed forearm implant was manufactured thicker than traditional trauma plate with minimum $3 \mathrm{~mm}$ or more. 
It is known that Ti6Al4V-ELI is used for extra strength and it definitely has superior physical properties than ordinary material. In case of screw, we tried to increase the fixation force of bone and plate by using a product with a diameter larger than the screw which is normally used for forearm. Therefore, it is truly believed that the screw pull-out after fixing it and the risk of plate and shaft fracture are lower or at least equal to those of existing products.

\section{Surgical procedures}

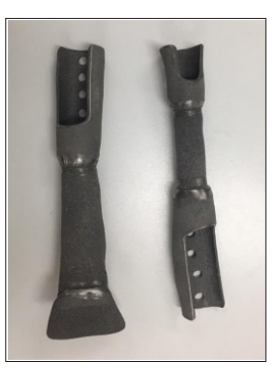

Figure 7: The 3D printed titanium forearm implants. (radius and ulna part).

In the supine position, a longitudinal incision on the volar side of forearm was performed. For radial and ulnar shaft, dissection was deepened through the plane between the brachioradialis muscle and flexor carpi radialis muscle. Pronator teres muscle was half-detached distally, and deep flexor muscles were preserved. By the described surgical approach distal ulna was not reached, therefore, additional incision was made on dorsal side of distal ulna. Totally four levels of osteotomy were cut with the cutting guide, and wide excision of the tumor was performed. The 3D-printed titanium implant was placed in the bone defect, and the fitting of the implant with the remained radius and ulna was grossly perfect. As planned preoperatively, four screws were inserted via premade holes on metal plates of implants for proximal radial and ulnar shaft for each. Since the distal ulnar remained bone was relatively short, only two screws were used for fixation. For distal radius, the implant has only volar side skirt for prevention of dorsal tendon

Table 1: Result of 3-point bending testing. abrasion. Original plan of the fixation for distal radius was using $\mathrm{x}$ shaped long cancellous screws, however screw fixation from ulnar side to radial side was impossible in real situation. Finally, one screw fixation from radial side to ulnar side and tension band wiring were performed. After fixation of implant to remained radius and ulna, the implant was secured and forearm rotation range was checked if it also got improved. During the postoperative period, we did not encounter any adverse situation (Figures 7 \& 8).

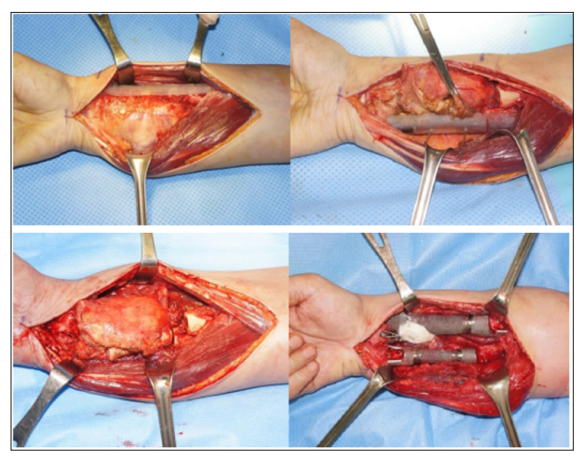

Figure 8: Images of the surgery process.

\section{Result}

\section{The Mechanical Property Test Results}

The mean maximum load was $14.18 \mathrm{kN} \pm 0.94 \mathrm{kN}$ and 3-point bending strength was $184.8 \mathrm{kN} \pm 23.3$ for ulna part. As for radius part, the mean maximum load was $19.27 \mathrm{kN} \pm 1.29 \mathrm{kN}$ and 3-point bending strength was $250.5 \mathrm{kN} \pm 25$.2. It is known that the average of the 3-point bending strength of the previous bone test data is $31.3 \mathrm{kN}$ for men and $15.5 \mathrm{kN}$ for women [11]. As a result of the experiment of 3D printed forearms, ulna part was about 5.9 times higher, whereas radius part was about 8 times higher than the aforementioned average scale. Mechanical testing results show that in all examples, despite shaft part is simply bent in ulna and radius part, shaft part is broken in one case of ulna (Table 1).

\begin{tabular}{|c|c|c|c|}
\hline Testing Specimen of Ulna & Maximum Load (k N) & 3-Point Bending Strength (k Nmm) & Failure Mode \\
\hline$\# 1$ & 15.12 & 226.8 & shaft were bent \\
\hline$\# 2$ & 13.41 & 167.7 & shaft were breakage \\
\hline$\# 3$ & 13.95 & 174.4 & shaft were bent \\
\hline$\# 4$ & 13.02 & 162.7 & shaft were bent \\
\hline$\# 5$ & 15.41 & 192.6 & shaft were bent \\
\hline Average & 14.18 & 184.8 & - \\
\hline Stdev & 0.94 & 23.3 & Failure Mode \\
\hline Testing Specimen of Radius & Maximum Load (k N) & 3-Point Bending Strength (k Nmm) & shaft were bent \\
\hline$\# 1$ & 19.29 & 289.4 & shaft were bent \\
\hline$\# 2$ & 18.91 & 236.4 & shaft were bent \\
\hline$\# 3$ & 19.12 & 239.1 & shaft were bent \\
\hline$\# 4$ & 17.51 & 218.9 & shaft were bent \\
\hline Average & 21.51 & 268.9 & - \\
\hline Stdev & 19.27 & 250.5 & - \\
\hline
\end{tabular}




\section{The clinical results}

Fortunately, there were no adverse postoperative conditions occurred. Intravenous antibiotics were administered for one week. The suction drain was removed for one week postoperatively, when the drainage was less than $5 \mathrm{cc}$. The antibiotic was switched to oral form for another week. After suture removal without wound complications two weeks postoperatively, the patient was allowed elbow and wrist motion in tolerable range. By the last follow-up at 12 months, potential complications, such as implant subluxation had not occurred. There was no pain around the surgical site. However, the range of motion of forearm rotation was similar to that before surgery and did not improve further (Figure 9).

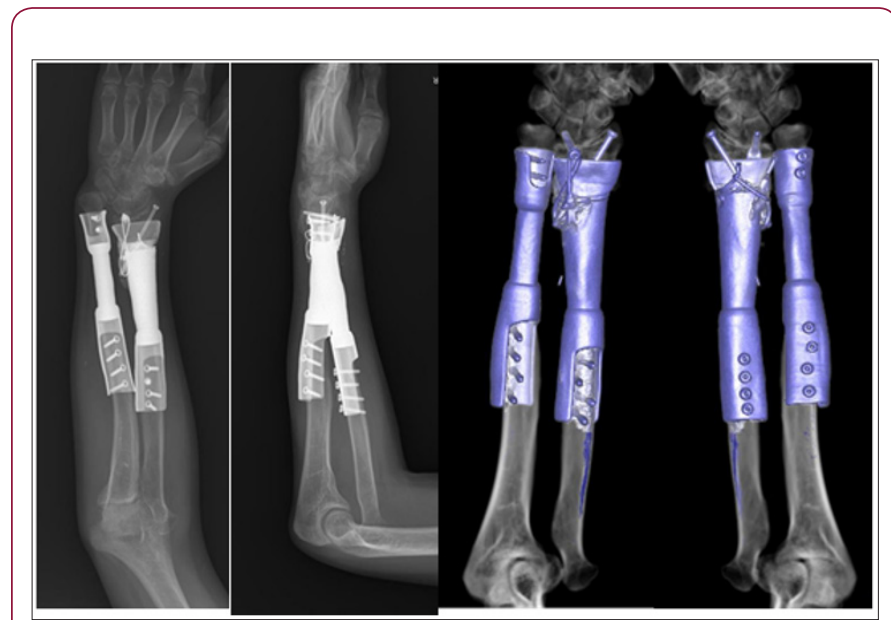

Figure 9: Images of Postoperative at the 12-Months.

\section{Discussion}

Throughout this study, we reconstruct 3D model using CT data of forearm tumor of the patient and simulated virtual surgery to set a safe margins of resection tumor. Then, we designed a method to stably fixation the modified 3D model with the existing bone and the 3D printed forearm implant. In addition, the final design was printed with 3D printing using Ti6Al4V-ELI material. Then, we verified the mechanical strength of the 3D printing implants. Finally, the patient was operated with the confirmed actual 3D printing forearm implants. Medical applications for 3D printing are expanding rapidly and about to revolutionize health care. [12-14] An application of the custom-made implant has many advantages in orthopedic oncology. A personalized implant can be made for every affected bone, regardless of the bone and tumor size. The perfect fit for the target patient results in more functional and anatomic reconstruction, and surgery can be performed without intraoperative fluoroscopicimaging. The duration of surgery time for reconstruction has been reduced in a gargantuan scale via personalized implant, and manufacturing period can also be reduced significantly.

In our patient, surgery was performed 2 weeks after the histologic diagnosis. Moreover, a 3D-printed custom-made implant is less costly than a traditional custom-made implant. Although 3D printer Patient-customized implants have many advantages, they have not yet been clinically proven for many years. As a result, there is insufficient verified performance as in the case of conventional commercial implants. However, in this study, we tried to compare the 3-point strength with the bone experimental data at least for shafts. One can find that this case report has some limitations. First, follow-up period was relatively shorter than usual. However, short term of follow-up was inevitable because limb salvage with 3D printed custom- made implant was a novel reconstruction option and the experience of this type of surgery needed to be announced and shared, since the authors thought that this case report of 3D-printed forearm implant surgery would use the longest followup.

Second, the hypothesis that long-term stability was achieved with soft tissue scarring on the mesh style implant surface could not be proved histologically in this report. Despite of absence of histologic analysis for peri-implant scarring due to ethical consideration, the special pain of the implanted the forearm can be moved was thought a collateral evidence for the hypothesis. In conclusion, 3D printing technology makes it possible to generate a patient-specific, biomechanically acceptable implant that can be fitted precisely to patient after tumor resection in orthopedic oncology.

\section{Acknowledgement}

This research was supported by a grant of the Korea Health Technology R\&D Project through the Korea Health Industry Development Institute (KHIDI), funded by the Ministry of Health \& Welfare, Republic of Korea (grant number : HI17C1823)

\section{References}

1. Baumgart R, Lenze U (2009) Expandable endoprostheses in malignant bone tumors in children: indications and limitations. Recent Results Cancer Res 179: 59-73.

2. DiCaprio MR, Friedlaender GE (2003) Malignant bone tumors: limb sparing versus amputation. J AmAcadOrthop Surg 11(1): 25-37.

3. Dotan A, Dadia S, Bickels J, Nirkin A, Flusser G, et al. (2010) Expandable endoprosthesis for limb-sparing surgery in children: long-term results. J Child Orthop 4(5): 391-400.

4. Finn HA, Simon MA (1991) Limb-salvage surgery in the treatment of osteosarcoma in skeletally immature individuals. Clin Orthop Relat Res 262: $108-118$

5. Nagarajan R, Neglia JP, Clohisy DR, Robison LL (2002) Limb salvage and amputation in survivors of pediatric lower-extremity bone tumors: what are the long-term implications? J Clin Oncol 20(22): 4493-4501.

6. Nystrom LM, Morcuende JA (2010) Expanding endoprosthesis for pediatric musculoskeletal malignancy: current concepts and results. Iowa Orthop J 30: 141-149.

7. Park DH, Jaiswal PK, Al Hakim W, Aston WJ, Pollock RC, et al. (2007) The use of massive endoprostheses for the treatment of bone metastases. Sarcoma 2007: 62151.

8. Saghieh S, Abboud MR, Muwakkit SA, Saab R, Rao B, et al. (2010) Sevenyear experience of using Repiphysis expandable prosthesis in children with bone tumors. Pediatr Blood Cancer 55(3): 457- 463.

9. Tang XD, Guo W, Yang RL, Yang Y, Ji T (2007) [Limb salvage surgery for osteosarcoma around the knee in children and adolescent patients]. Zhonghua Wai Ke Za Zhi 45(10): 669-672.

10. Ferguson PC, Zdero R, Schemitsch EH, Deheshi BM, Bell RS, et al. (2011) A biomechanical evaluation of press-fit stem constructs for tumor endoprosthetic reconstruction of the distal femur. J Arthroplasty 26(8): 1373-1379. 
11. Eva Maria Lochmu Ller, Christoph A Lill, Volker Kuhn, Erich Schneider, Felix Eckstein (2002) Radius Bone Strength in Bending, Compression, and Falling and Its Correlation with Clinical Densitometry at Multiple Sites. Journal Of Bone And Mineral Research17(9): 1629-1638.

12. Cui H, Nowicki M, Fisher JP, Zhang LG (2016) 3D bioprinting for organ regeneration. Adv Healthc Mater 6(1).

ISSN: 2574-1241

DOI: $10.26717 / B J S T R .2018 .10 .001950$

Hyun Guy Kang. Biomed J Sci \& Tech Res

CC (P) This work is licensed under Creative Commons Attribution 4.0 License

Submission Link: https://biomedres.us/submit-manuscript.php
13. Zopf DA, Hollister SJ, Nelson ME, Ohye RG, Green GE (2013) Bioresorbable airway splint created with a three-dimensional printer. N Engl J Med 368(21): 2043-2045.

14. Ventola CL (2014) Medical applications for 3D printing: current and projected uses. P T 39(10): 704-711.

$\begin{array}{ll}\text { BIOMEDICAL } & \text { Assets of Publishing with us } \\ \text { RESEARCHES } & \text { - Global archiving of articles } \\ \text { - Immediate, unrestricted online access }\end{array}$

